\title{
AXISYMMETRIC SHELL MODEL USING A THREE-FIELD DUAL-MIXED VARIATIONAL PRINCIPLE
}

\author{
BALÁZs TÓTH \\ Department of Mechanics, University of Miskolc \\ 3515 Miskolc-Egyetemváros, Hungary \\ mechtb@uni-miskolc.hu
}

[Received: April 10, 2007]

\begin{abstract}
A dimensionally reduced axisymmetric shell model using a three-field complementary energy-based variational principle set up in terms of non-symmetric stresses, rotations and displacements is derived. Considering axisymmetrically loaded shells of revolution, a special form of the three-dimensional variational principle of Hellinger-Reissner is obtained and applied for the derivation of the Euler-Lagrange equations and natural boundary conditions of the shell model.
\end{abstract}

Mathematical Subject Classification:

Keywords: axisymmetric shell, three-field dual-mixed variational principle, dimensional reduction, Euler-Lagrange equations

\section{INTRODUCTION}

Mixed variational principles in elasticity offer the possibility of approximating the stress space directly. Complementary energy-based dual-mixed variational formulations and properly designed finite element models have the additional advantage that they provide better convergence rates and higher accuracy for the variables of primary interest, i.e. for the stresses, when compared to strain energy-based primalmixed formulations and displacement-based formulations. Stress-based finite element models, furthermore, are known to be free from those numerical locking problems that appear in many displacement-based finite element models. These facts seem to be especially important for shell models and shell finite elements for which the modelling and approximation difficulties can lead to several type of numerical problems.

The main objective of this paper is to derive a dimensionally reduced axisymmetric shell model based on Hellinger-Reissner's three-field dual-mixed variational principle. Its functional can be derived from the total complementary energy functional

$$
K_{d}\left(\sigma^{r s}\right)=-\frac{1}{2} \int_{(V)} \sigma^{k l} \varepsilon_{k l}\left(\sigma^{r s}\right) \mathrm{d} V+\int_{\left(S_{u}\right)} \tilde{u}_{k} \sigma^{k l} n_{l} \mathrm{~d} S,
$$


which depends on the stress tensor $\sigma^{r s}$ alone. Here $V$ denotes the volume of the body in the undeformed configuration, $\varepsilon_{k l}$ is the symmetric strain tensor and $\widetilde{u}_{k}$ is the displacement vector prescribed on the surface part $S_{u}$ with outward unit normal $n_{l}$. The subsidiary conditions to the total complementary energy functional 1.1$)$ are the equilibrium equations

$$
\sigma_{; q}^{p q}+b^{p}=0 \quad x^{m} \in V,
$$

where $b^{p}$ stands for the body forces, the symmetry condition

$$
\epsilon_{p q r} \sigma^{p q}=0 \quad x^{m} \in V,
$$

where $\epsilon_{p q r}$ is the covariant permutation tensor, and the stress boundary conditions

$$
\widetilde{p}^{k}=\sigma^{k l} n_{l} \quad x^{m} \in S_{p},
$$

where $\widetilde{p}^{k}$ are prescribed surface tractions on $S_{p}$ with outward unit normal $n_{l}$ (the surface of the body is $\left.S=S_{p} \cup S_{u}, S_{p} \cap S_{u}=\emptyset\right)$. For linear elastic materials the strain tensor $\varepsilon_{k l}$ can be obtained from the inverse stress-strain relations (Hooke's law)

$$
\varepsilon_{k l}=C_{k l r s}^{-1} \sigma^{r s} \quad x^{m} \in V,
$$

where the fourth-order tensor $C_{k l r s}^{-1}$ with symmetry properties $C_{k l r s}^{-1}=C_{k l s r}^{-1}=C_{r s k l}^{-1}$ is the elastic compliance tensor.

Classical, complementary energy-based and dimensionally reduced shell models in terms of symmetric stresses can be derived using functional (1.1). In that case both the translational and rotational equilibrium equations, $(1.2)$ and $(1.3)$, as well as the stress boundary conditions (1.4) should be satisfied a priori. A dimensionally reduced cylindrical shell model based on this approach using second-order stress functions can be found in 3 . The related finite element formulation requires, however, $C_{1}$ continuous approximation of the second-order stress functions and this requirement, which is primarily due to the symmetry condition for the stress tensor, makes it rather difficult to establish a numerically efficient formulation for general shells.

One possibility to overcome the difficulties mentioned in connection with the derivation of numerically efficient stress-based shell models is the incorporation of the symmetry condition for the stress tensor into the principle by the use of the rotations as Lagrangian multipliers. A dimensionally reduced non-linear shell model using the corresponding dual-mixed variational principle of Fraeijs de Veubeke [6] has been derived in [4].

Another possibility for the derivation of dimensionally reduced stress-based shell models is to apply the three-field dual-mixed variational principle of Hellinger-Reissner [7, 9, 10. The functional of this principle can be considered as the sum of the total complementary energy functional (1.1) and two Lagrangian multiplier terms which red ensure the fulfillment of the translational and rotational equilibrium equations, (1.2) and (1.3). In the linear theory of elasticity the functional red is of the form

$$
H R_{d}\left(\sigma^{r s}, \phi^{s}, u_{p}\right)=-\int_{(V)} W_{c}\left(\sigma^{r s}\right) \mathrm{d} V+\int_{\left(S_{u}\right)} \widetilde{u}_{p} \sigma^{p q} n_{q} \mathrm{~d} S+
$$




$$
+\int_{(V)} \sigma^{p q} \epsilon_{p q s} \phi^{s} \mathrm{~d} V-\int_{(V)} u_{p}\left(\sigma_{; q}^{p q}+b^{p}\right) \mathrm{d} V,
$$

where $\sigma^{r s}$ is the not a priori symmetric stress tensor, red the strain tensor $\varepsilon_{p q}$ is a symmetric one, $\phi^{s}$ denotes the axial vector of the skew-symmetric rotation tensor, $\psi_{p q}$ red which are related to each other via the equation

$$
\psi_{p q}=-\epsilon_{p q s} \phi^{s},
$$

$\widetilde{u}_{p}$ is the prescribed displacement vector on $S_{u}$ with outward unit normal $n_{q}$ and $W_{c}$ is the complementary strain energy density defined by

$$
W_{c}\left(\sigma^{r s}\right)=\frac{1}{2} \sigma^{p q} \varepsilon_{p q}\left(\sigma^{r s}\right)=\frac{1}{2} C_{p q r s}^{-1} \sigma^{p q} \sigma^{r s} .
$$

The only subsidiary conditions to be satisfied by functional (1.6) are the stress boundary conditions (1.4). It means that applying Hellinger-Reissner's dual-mixed principle with functional (1.6), a priori satisfaction of neither the translational nor the rotational equilibrium equations is required.

It is noted here that the classical two-field dual-mixed variational principle of Hellinger-Reissner with a priori symmetric stresses can also be used as a starting point for deriving dimensionally reduced stress-based shell models. The design of stable and efficient finite element models assuming symmetric stresses is, however, much more difficult than that with not a priori symmetric ones (for a discussion of the latter problem, see [5]).

This paper presents a dimensional reduction procedure for shells of revolution applying the three-dimensional dual-mixed variational principle of Hellinger-Reissner with functional (1.6). Section 2 contains the geometric description of the shell of revolution. In section 3 the three-dimensional equilibrium equations are derived in the curvilinear coordinate system attached to the shell middle surface. The dimensional reduction procedure is presented in Section 4. Applying truncated power series expansions, the independent variables, i.e., the stresses, the displacements and the rotations are approximated by polynomials of the first- and second-order in the thickness coordinate. After inserting the variables expanded into series into the special form of the Hellinger-Reissner functional for thin shells of revolution and introducing the notions of strain and rotation resultants as well as that of the prescribed displacement resultants, the Euler-Lagrange equations and the natural boundary conditions of the dimensionally reduced shell model are derived, assuming axisymmetrical loads. Section 5 contains some concluding remarks and an outlook for further research in the presented direction.

\section{Geometry of the Shell of Revolution}

Let $x^{k}$ refer to a fixed right-handed orthogonal Cartesian frame in an Euclidean threedimensional space, the corresponding base vectors are denoted by $\mathbf{e}_{k}$. We consider a shell of revolution as a three-dimensional body, the rotation axis of which is defined 
by

$$
0 \leq x^{1} \leq L,
$$

where $L$ is the length of the shell (see Figure 1). The axisymmetric shell is a $3 \mathrm{D}$

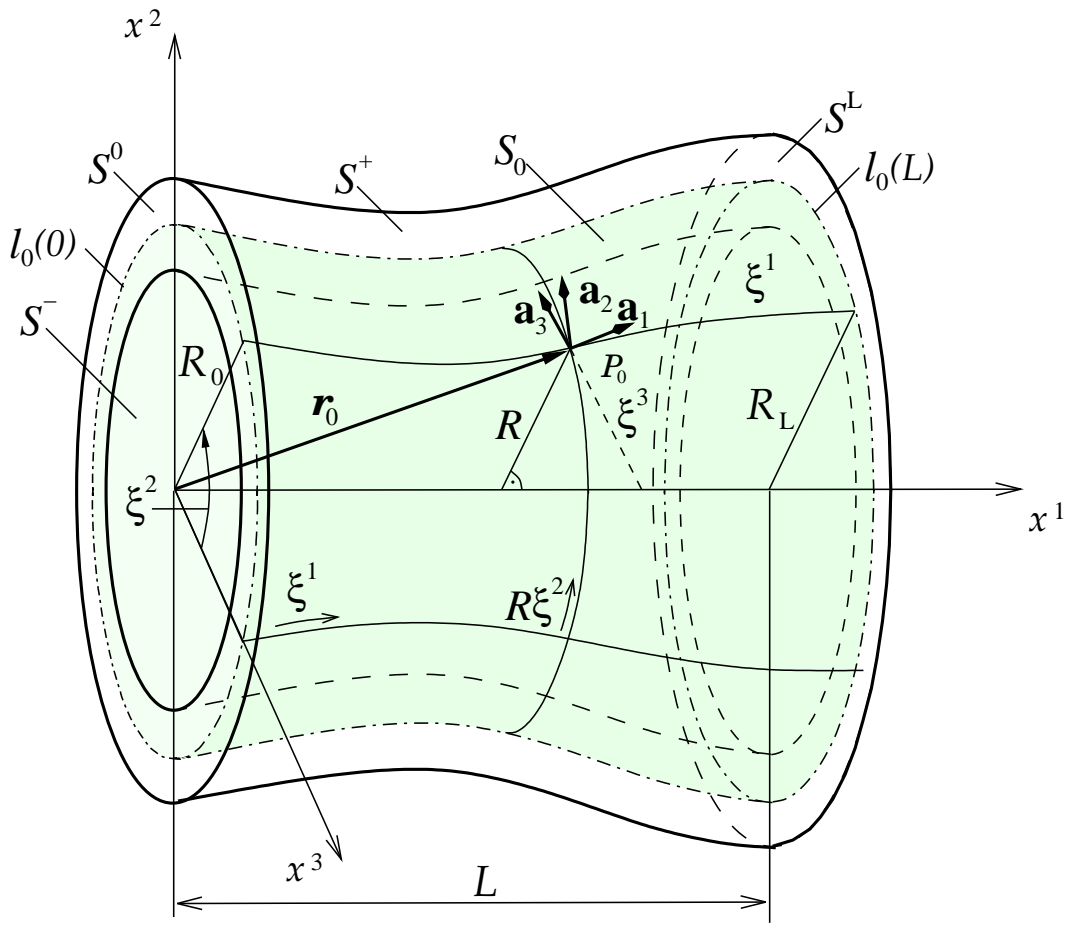

Figure 1. Axisymmetric shell

region bounded by two axisymmetric surfaces $S^{+}$and $S^{-}$called the top and bottom surfaces which are symmetrically situated with respect to the surface $S_{0}$ called the middle surface, and the lateral surfaces $S^{0}$ and $S^{L}$, the intersections of which with $S_{0}$ are the circles $\ell_{0}(0)$ and $\ell_{0}(L)$, respectively (see Figure 1). The middle surface of the shell can be generated by rotating a curve about the $x^{1}$-axis. This curve is the meridian curve defined by the function

$$
R=R\left(x^{1}\right)>0 .
$$

The shell is parametrized by the curvilinear coordinates $\xi^{m}$, with $\xi^{\alpha}$ called surface coordinates on $S_{0}$ and with $\xi^{3}$ which is the arc-length measured along the normal to the middle surface $\left(\xi^{3}=0\right)$. Throughout this paper, the usual summation convention is used and the range of the Latin indices is 1,2,3 and that of the Greek indices is 1 and 2. The undeformed configuration of the shell is defined by

$$
V=\left\{\xi^{m}\left|\xi^{\alpha} \in S_{0},\right| \xi^{3} \mid \leqslant \frac{d}{2}\right\}
$$


where $d$, the distance between $S^{+}$and $S^{-}$measured along $\xi^{3}$, is the thickness of the shell. Henceforward it will be assumed that $d$ is constant. The boundary of $V$, denoted by $S$, consists of the top and bottom surfaces

$$
S^{ \pm}=\left\{\xi^{m} \mid \xi^{\alpha} \in S_{0}, \xi^{3}= \pm \frac{d}{2}\right\}
$$

and lateral surfaces

$$
\begin{aligned}
S^{0} & =\left\{\xi^{m}\left|\xi^{\alpha} \in \ell_{0}(0)\right| \xi^{3} \mid \leq \frac{d}{2}\right\}, \\
S^{L} & =\left\{\xi^{m}\left|\xi^{\alpha} \in \ell_{0}(L),\right| \xi^{3} \mid \leq \frac{d}{2}\right\} .
\end{aligned}
$$

The relation between the Cartesian coordinates $x^{k}$ and the curvilinear coordinates $\xi^{\alpha}$ parametrizing the shell middle surface $S_{0}$ is given by

$$
\begin{aligned}
& x^{1}=f\left(\xi^{1}\right), \\
& x^{2}=R \sin \xi^{2}, \\
& x^{3}=R \cos \xi^{2},
\end{aligned}
$$

where $\xi^{1}$ is measured along the meridian curve and $\xi^{2}$ is the polar angle:

$$
0 \leq \xi^{2}<2 \pi
$$

measured along the latitude circles lying in the planes perpendicular to the rotation axis (see Figure 1). The position vector of an arbitrary point $P_{0} \in S_{0}$ can be written in the Cartesian coordinate frame as

$$
\mathbf{r}_{0}=x^{k} \mathbf{e}_{k}=x^{1} \mathbf{e}_{1}+R \sin \xi^{2} \mathbf{e}_{2}+R \cos \xi^{2} \mathbf{e}_{3} .
$$

Introducing the notations

$$
A_{1}\left(x^{1}\right)=\frac{\mathrm{d} \xi^{1}}{\mathrm{~d} x^{1}}, \quad \frac{1}{A_{1}\left(x^{1}\right)}=\frac{\mathrm{d} x^{1}}{\mathrm{~d} \xi^{1}},
$$

the base vectors $\mathbf{a}_{\alpha}$ of the surface coordinate system can be obtained by deriving the position vector $\mathbf{r}_{0} 2.11$ with respect to $\xi^{\alpha}$ :

$$
\begin{aligned}
& \mathbf{a}_{1}=\frac{\partial \mathbf{r}_{0}}{\partial \xi^{1}}=\frac{1}{A_{1}} \mathbf{e}_{1}+\frac{1}{A_{1}} R^{\prime} \sin \xi^{2} \mathbf{e}_{2}+\frac{1}{A_{1}} R^{\prime} \cos \xi^{2} \mathbf{e}_{3}=\mathbf{a}^{1}, \\
& \mathbf{a}_{2}=\frac{\partial \mathbf{r}_{0}}{\partial \xi^{2}}=R \cos \xi^{2} \mathbf{e}_{2}-R \sin \xi^{2} \mathbf{e}_{3}=R^{2} \mathbf{a}^{2} .
\end{aligned}
$$

The base vector $\mathbf{a}_{3}$, which is a normal to the middle surface $S_{0}$, is obtained as

$$
\mathbf{a}_{3}=\frac{\mathbf{a}_{1} \times \mathbf{a}_{2}}{\left|\mathbf{a}_{1} \times \mathbf{a}_{2}\right|}=\frac{1}{A_{1}}\left(\sin \xi^{2} \mathbf{e}_{2}+\cos \xi^{2} \mathbf{e}_{3}-R^{\prime} \mathbf{e}_{1}\right)=\mathbf{a}^{3},
$$

where the prime in the superscript indicates differentiation with respect to the coordinate $x^{1}$. Making use of equations 2.13)-2.15), the matrices of the covariant and contravariant metric tensor on the middle surface are obtained as

$$
\left[a_{k \ell}\right]=\left[\begin{array}{ccc}
1 & 0 & 0 \\
0 & R^{2} & 0 \\
0 & 0 & 1
\end{array}\right], \quad\left[a^{p q}\right]=\left[\begin{array}{ccc}
1 & 0 & 0 \\
0 & \frac{1}{R^{2}} & 0 \\
0 & 0 & 1
\end{array}\right] .
$$


The covariant base vectors at an arbitrary shell point $P$ are denoted by $\mathbf{g}_{l}$ (see Figure 2). The covariant metric tensor at $P$ is denoted by $g_{k l}\left(g_{3 \beta}=0\right)$. The Christoffel

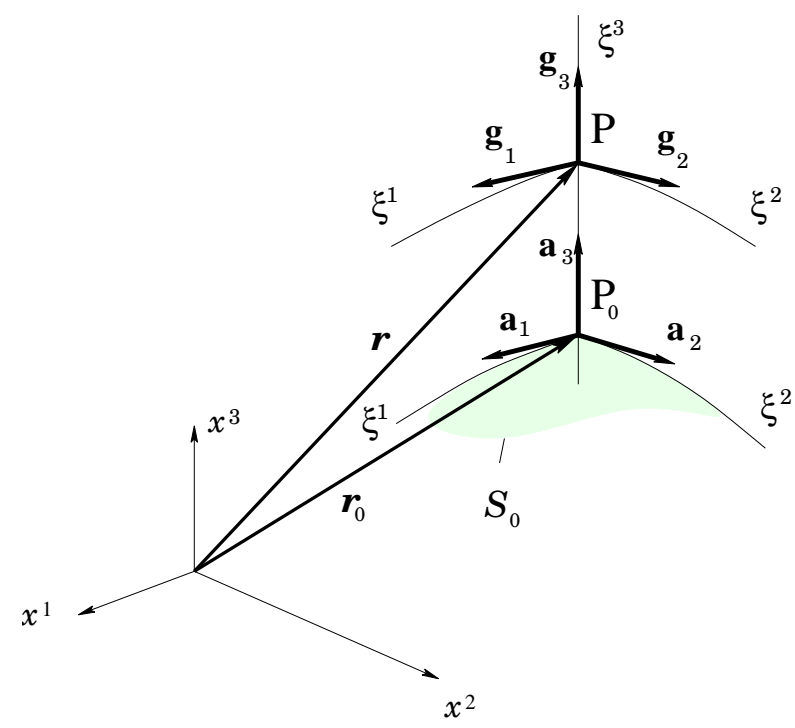

Figure 2. Local coordinate systems and their base vectors

symbols of the second kind on the middle surface $S_{0}$ are defined by the equations

$$
\bar{\Gamma}_{s m}^{k}=\mathbf{a}_{s, m} \cdot \mathbf{a}^{k}, \quad \bar{\Gamma}_{\alpha \beta}^{\kappa}=\mathbf{a}_{\alpha, \beta} \cdot \mathbf{a}^{\kappa}, \quad \bar{\Gamma}_{\lambda 3}^{\mu}=\bar{\Gamma}_{33}^{m}=\bar{\Gamma}_{3 \kappa}^{3}=\bar{\Gamma}_{\lambda 3}^{3}=0,
$$

where the subscripts $m$ or $\beta$ preceded by a coma mean partial differentiation with respect to the corresponding coordinates $\xi^{m}$ or $\xi^{\beta}$. The curvature tensor of the middle surface is given by

$$
b_{\kappa \lambda}=\bar{\Gamma}_{\kappa \lambda}^{3}, \quad b_{\kappa}^{\mu}=\bar{\Gamma}_{3 \kappa}^{\mu} .
$$

The relation between the covariant and contravariant base vectors at $P$ and $P_{0} \in S_{0}$ is written in the following form:

$$
\mathbf{g}_{l}=\mu_{l}^{k} \mathbf{a}_{k}, \quad \mathbf{g}^{m}=\left(\mu^{-1}\right)_{p}^{m} \mathbf{a}^{p},
$$

where $\mu_{l}^{k}$ and $\left(\mu^{-1}\right)_{p}^{m}$ are, respectively, the shifter and the inverse shifter. Making use of 2.19) an arbitrary tensor $\mathbf{T}$ defined at the point $P$ of the shell can be shifted to the point $P_{0}$ of the middle surface:

$$
\mathbf{T}=T_{k}^{\ell} \mathbf{g}^{k} \mathbf{g}_{l}=\bar{T}_{m}^{n} \mathbf{a}^{m} \mathbf{a}_{n},
$$

where a bar over a tensor variable denotes its tensorial components related to the base vectors $\mathbf{a}_{k}$ of the shell middle surface, and

$$
\bar{T}_{m}^{n}=T_{k}^{\ell}\left(\mu^{-1}\right)_{m}^{k} \mu_{\ell}^{n}
$$


are the shifted tensor components. Thus the covariant derivative tensor $T_{; m}^{p q}$ can be obtained by

where

$$
T_{; m}^{p q}=\bar{T}_{\mid m}^{k l}\left(\mu^{-1}\right)_{k}^{p}\left(\mu^{-1}\right)_{l}^{q},
$$

$$
\bar{T}_{\mid m}^{k l}=\bar{T}_{, m}^{k l}+\bar{\Gamma}_{s m}^{k} \bar{T}^{s l}+\bar{\Gamma}_{r m}^{l} \bar{T}^{k r}
$$

is the three-dimensional covariant derivative on the middle surface $S_{0}$. The covariant derivative 2.23) can be separated into

$$
\bar{T}_{\mid \nu}^{k l}=\bar{T}_{, \nu}^{k l}+\bar{\Gamma}_{s \nu}^{k} \bar{T}^{s l}+\bar{\Gamma}_{r \nu}^{l} \bar{T}^{k r}
$$

and

$$
\bar{T}_{\mid 3}^{k l}=\bar{T}_{, 3}^{k l} .
$$

In the case of axisymmetric shells the non-zero components of the Christoffel symbols 2.17 and the curvature tensor 2.18 are, respectively,

$$
\bar{\Gamma}_{12}^{2}=\bar{\Gamma}_{21}^{2}=\frac{R^{\prime}}{R A_{1}}, \quad \bar{\Gamma}_{22}^{1}=-\frac{R R^{\prime}}{A_{1}}
$$

and

$$
\begin{gathered}
b_{11}=\bar{\Gamma}_{11}^{3}=\frac{R^{\prime \prime}}{A_{1}^{3}}, \quad b_{22}=\bar{\Gamma}_{22}^{3}=-\frac{R}{A_{1}}, \\
b_{1}^{1}=-\frac{1}{R_{1}}=-\bar{\Gamma}_{31}^{1}=\frac{R^{\prime \prime}}{A_{1}^{3}}, \quad b_{2}^{2}=-\frac{1}{R_{2}}=-\bar{\Gamma}_{32}^{2}=-\frac{1}{R A_{1}},
\end{gathered}
$$

where $R_{1}$ and $R_{2}$ are the principal radii of curvature of the middle surface $S_{0}$. In view of 2.28, the matrix of the shifter 2.19 can be represented by

$$
\left[\mu_{l}^{k}\right]=\left[\begin{array}{ccc}
1-\frac{R^{\prime \prime}}{A_{1}^{3}} \xi^{3} & 0 & 0 \\
0 & 1+\frac{1}{R A_{1}} \xi^{3} & 0 \\
0 & 0 & 1
\end{array}\right] .
$$

For thin shells, the thickness is assumed to be small, i.e., the relations

$$
\frac{d}{L} \ll 1, \quad \frac{d}{R_{\min }} \ll 1
$$

hold true, where

$$
R_{\min }=\min _{x^{1} \in[0 ; L]}\left\{\left|R_{1}\right|,\left|R_{2}\right|\right\},
$$

thus

$$
\mu_{l}^{k} \cong\left(\mu^{-1}\right)_{l}^{k} \cong \delta_{l}^{k}, \mu=1,
$$

where $\mu$ is the determinant of the shifter 2.29 . For thin shells of revolution, the volume element $\mathrm{d} V$ and the surface elements $\mathrm{d} S^{0}$ and $\mathrm{d} S^{L}$ are given by

where

$$
\begin{gathered}
\mathrm{d} V=R \mathrm{~d} \xi^{1} \mathrm{~d} \xi^{2} \mathrm{~d} \xi^{3}, \\
\mathrm{~d} S^{0}=R_{0} \mathrm{~d} \xi^{2} \mathrm{~d} \xi^{3} \\
\mathrm{~d} S^{L}=R_{L} \mathrm{~d} \xi^{2} \mathrm{~d} \xi^{3}
\end{gathered}
$$

$$
R=R\left(x^{1}=0\right)=R_{0}, \quad R=R\left(x^{1}=L\right)=R_{L} .
$$




\section{EqUiLIBRIUM EQUATIONS OF SHELLS OF REVOLUTION}

The three-dimensional equilibrium equations of the axisymmetric shells are derived from equations $(1.2)$. Using the derivation rule $(2.22)$ and the geometric properties $2.30-2.32$, the three-dimensional translational equilibrium equations for thin shells can be written in the following form:

$$
\bar{\sigma}_{\mid l}^{p l}+\bar{b}^{p}=0 \quad \xi^{m} \in V .
$$

Making use of equations 2.26 - 2.28 obtained for the Christoffel symbols, the threedimensional scalar equilibrium equations (3.1) can be written as

$$
\begin{aligned}
& \bar{\sigma}_{, 1}^{11}-\frac{R^{\prime \prime}}{A_{1}^{3}}\left(\bar{\sigma}^{31}+\bar{\sigma}^{13}\right)+\bar{\sigma}_{, 2}^{12}+\frac{1}{R A_{1}}\left(R^{\prime} \bar{\sigma}^{11}+\bar{\sigma}^{13}\right)-\frac{R R^{\prime}}{A_{1}} \bar{\sigma}^{22}+\bar{\sigma}_{, 3}^{13}+\bar{b}^{1}=0, \\
& \bar{\sigma}_{, 1}^{21}-\frac{R^{\prime \prime}}{A_{1}^{3}} \bar{\sigma}^{23}+\bar{\sigma}_{, 2}^{22}+\frac{R^{\prime}}{R A_{1}}\left(\bar{\sigma}^{12}+2 \bar{\sigma}^{21}\right)+\frac{1}{R A_{1}}\left(\bar{\sigma}^{23}+\bar{\sigma}^{32}\right)+\bar{\sigma}_{, 3}^{23}+\bar{b}^{2}=0 \\
& \bar{\sigma}_{, 1}^{31}+\frac{R^{\prime \prime}}{A_{1}^{3}}\left(\bar{\sigma}^{11}-\bar{\sigma}^{33}\right)+\bar{\sigma}_{, 2}^{32}+\frac{1}{R A_{1}}\left(R^{\prime} \bar{\sigma}^{31}+\bar{\sigma}^{33}\right)-\frac{R}{A_{1}} \bar{\sigma}^{22}+\bar{\sigma}_{, 3}^{33}+\bar{b}^{3}=0 .
\end{aligned}
$$

For axisymmetrically loaded shells of revolution the differentiation with respect to $\xi^{2}$ leads to zero, thus the equations $3.2-3.4$ can be simplified to

$$
\begin{gathered}
\bar{\sigma}_{, 1}^{11}-\frac{R^{\prime \prime}}{A_{1}^{3}}\left(\bar{\sigma}^{31}+\bar{\sigma}^{13}\right)+\frac{1}{R A_{1}}\left(R^{\prime} \bar{\sigma}^{11}+\bar{\sigma}^{13}\right)-\frac{R R^{\prime}}{A_{1}} \bar{\sigma}^{22}+\bar{\sigma}_{, 3}^{13}+\bar{b}^{1}=0, \\
\bar{\sigma}_{, 1}^{21}-\frac{R^{\prime \prime}}{A_{1}^{3}} \bar{\sigma}^{23}+\frac{R^{\prime}}{R A_{1}}\left(\bar{\sigma}^{12}+2 \bar{\sigma}^{21}\right)+\frac{1}{R A_{1}}\left(\bar{\sigma}^{23}+\bar{\sigma}^{32}\right)+\bar{\sigma}_{, 3}^{23}+\bar{b}^{2}=0 \\
\bar{\sigma}^{31}{ }_{, 1}+\frac{R^{\prime \prime}}{A_{1}^{3}}\left(\bar{\sigma}^{11}-\bar{\sigma}^{33}\right)+\frac{1}{R A_{1}}\left(R^{\prime} \bar{\sigma}^{31}+\bar{\sigma}^{33}\right)-\frac{R}{A_{1}} \bar{\sigma}^{22}+\bar{\sigma}_{, 3}^{33}+\bar{b}^{3}=0 .
\end{gathered}
$$

These two-dimensional scalar equilibrium equations can be separated into two independent groups according to the stress components. Equations (3.5) and (3.7) involve the stress components $\bar{\sigma}^{11}, \bar{\sigma}^{22}, \bar{\sigma}^{13}, \bar{\sigma}^{31}$ and $\bar{\sigma}^{33}$ which describe the bending-shearing problems (including tension-compression) of the axisymmetric shell while equation (3.6) describes the torsion problem of the shell. The corresponding stress components are $\bar{\sigma}^{12}, \bar{\sigma}^{21}, \bar{\sigma}^{23}$ and $\bar{\sigma}^{32}$.

\section{Dimensionally Reduced aXisymmetric Shell MOdel}

4.1. Approximation of the stress components along the thickness. Let us consider a shell of revolution loaded axisymmetrically. Then the problem is a two dimensional one - each quantity depends on $\xi^{1}$ and $\xi^{3}$ only. The components of the tensor variables are assumed to be shifted to the points of the middle surface. The fundamental variables of the two-dimensional problems can be expanded in power series with respect to the thickness coordinate $\xi^{3}$. After being expanded into series 
the stresses $\bar{\sigma}^{k l}$, the rotation components $\bar{\phi}^{m}$ and the displacements $\bar{u}_{n}$ can be written as

$$
\begin{aligned}
\bar{\sigma}^{k \ell}\left(\xi^{1}, \xi^{3}\right) & =\sum_{i=0}^{\infty}{ }_{i} \bar{\sigma}^{k \ell}\left(\xi^{1}\right)\left(\xi^{3}\right)^{i} \\
\bar{\phi}^{m}\left(\xi^{1}, \xi^{3}\right) & =\sum_{i=0}^{\infty}{ }_{i} \bar{\phi}^{m}\left(\xi^{1}\right)\left(\xi^{3}\right)^{i} \\
\bar{u}_{n}\left(\xi^{1}, \xi^{3}\right) & =\sum_{i=0}^{\infty}{ }_{i} \bar{u}_{n}\left(\xi^{1}\right)\left(\xi^{3}\right)^{i}
\end{aligned}
$$

where the rotation coefficients ${ }_{i} \bar{\phi}^{m}\left(\xi^{1}\right)$, the stress coefficients ${ }_{i} \bar{\sigma}^{k \ell}\left(\xi^{1}\right)$ and the displacement coefficients ${ }_{i} \bar{u}_{n}\left(\xi^{1}\right)$ depend only on the surface coordinate $\xi^{1}$. Substituting stress components (4.1) into the two-dimensional scalar equilibrium equations (3.5)-3.7) and making separation with respect to the powers of the thickness coordinate $\xi^{3}$, the following one-dimensional equilibrium equations are obtained for the one-dimensional stress coefficients:

$$
\begin{array}{r}
{ }_{i} \bar{\sigma}_{, 1}^{11}-\frac{R^{\prime \prime}}{A_{1}^{3}}\left({ }_{i} \bar{\sigma}^{31}+{ }_{i} \bar{\sigma}^{13}\right)+\frac{1}{R A_{1}}\left(R^{\prime}{ }_{i} \bar{\sigma}^{11}+{ }_{i} \bar{\sigma}^{13}\right)-\frac{R R^{\prime}}{A_{1}}{ }_{i} \bar{\sigma}^{22}+ \\
+(i+1){ }_{i+1} \bar{\sigma}^{13}+{ }_{i} \bar{b}^{1}=0, \quad i=0,1,2, \ldots, \\
{ }_{i} \bar{\sigma}^{21}{ }_{, 1}-\frac{R^{\prime \prime}}{A_{1}^{3}}{ }_{i} \bar{\sigma}^{23}+ \\
\left.+(i+1){ }_{i+1} \bar{R}^{\prime} A_{1}{ }_{i} \bar{\sigma}^{12}+2{ }_{i} \bar{\sigma}^{21}\right)+\frac{1}{R A_{1}}\left({ }_{i} \bar{\sigma}^{23}+{ }_{i} \bar{\sigma}^{32}\right)+ \\
+(i=0,1,2, \ldots, \\
{ }_{i} \bar{\sigma}^{31}{ }_{, 1}+\frac{R^{\prime \prime}}{A_{1}^{3}}\left({ }_{i} \bar{\sigma}^{11}-{ }_{i} \bar{\sigma}^{33}\right)+\frac{1}{R A_{1}}\left(R^{\prime}{ }_{i} \bar{\sigma}^{31}+{ }_{i} \bar{\sigma}^{33}\right)-\frac{R}{A_{1}}{ }_{i} \bar{\sigma}^{22}+ \\
+(i+1){ }_{i+1} \bar{\sigma}^{33}+{ }_{i} \bar{b}^{3}=0, \quad i=0,1,2, \ldots .
\end{array}
$$

Depending on the number of equations selected from the above equilibrium equations, a large number of dimensionally reduced axisymmetric shell models can be derived. In this paper a shell model, which is based on the equations valid for $i=0$ and $i=1$, is investigated assuming that the higher-order equilibrium equations are identically satisfied. In this case the stress components $\bar{\sigma}^{k \lambda}$ and $\bar{\sigma}^{k 3}$ are approximated, respectively, by polynomials of the first- and second-degree in $\xi^{3}$ :

$$
\begin{aligned}
& \bar{\sigma}^{k \lambda}\left(\xi^{1}, \xi^{3}\right)={ }_{0} \bar{\sigma}^{k \lambda}\left(\xi^{1}\right)+{ }_{1} \bar{\sigma}^{k \lambda}\left(\xi^{1}\right) \xi^{3} \\
& \bar{\sigma}^{k 3}\left(\xi^{1}, \xi^{3}\right)={ }_{0} \bar{\sigma}^{k 3}\left(\xi^{1}\right)+{ }_{1} \bar{\sigma}^{k 3}\left(\xi^{1}\right) \xi^{3}+{ }_{2} \bar{\sigma}^{k 3}\left(\xi^{1}\right)\left(\xi^{3}\right)^{2} .
\end{aligned}
$$

The corresponding one-dimensional translational equilibrium equations for the 18 stress coefficients ${ }_{0} \bar{\sigma}^{k \lambda},{ }_{1} \bar{\sigma}^{k \lambda},{ }_{0} \bar{\sigma}^{k 3},{ }_{1} \bar{\sigma}^{k 3},{ }_{2} \bar{\sigma}^{k 3}$ are obtained from equations (4.7) (4.8):

$$
{ }_{0} \bar{\sigma}_{, 1}^{11}-\frac{R^{\prime \prime}}{A_{1}^{3}}\left({ }_{0} \bar{\sigma}^{31}+{ }_{0} \bar{\sigma}^{13}\right)+\frac{1}{R A_{1}}\left(R^{\prime}{ }_{0} \bar{\sigma}^{11}+{ }_{0} \bar{\sigma}^{13}\right)-\frac{R R^{\prime}}{A_{1}}{ }_{0} \bar{\sigma}^{22}+{ }_{1} \bar{\sigma}^{13}+{ }_{0} \bar{b}^{1}=0
$$




$$
\begin{aligned}
& { }_{0} \bar{\sigma}_{, 1}^{21}-\frac{R^{\prime \prime}}{A_{1}^{3}}{ }_{0} \bar{\sigma}^{23}+\frac{R^{\prime}}{R A_{1}}\left({ }_{0} \bar{\sigma}^{12}+2_{0} \bar{\sigma}^{21}\right)+\frac{1}{R A_{1}}\left({ }_{0} \bar{\sigma}^{23}+{ }_{0} \bar{\sigma}^{32}\right)+{ }_{1} \bar{\sigma}^{23}+{ }_{0} \bar{b}^{2}=0, \quad(4.10) \\
& { }_{0} \bar{\sigma}^{31}{ }_{, 1}+\frac{R^{\prime \prime}}{A_{1}^{3}}\left({ }_{0} \bar{\sigma}^{11}-{ }_{0} \bar{\sigma}^{33}\right)+\frac{1}{R A_{1}}\left(R^{\prime}{ }_{0} \bar{\sigma}^{31}+{ }_{0} \bar{\sigma}^{33}\right)-\frac{R}{A_{1}}{ }_{0} \bar{\sigma}^{22}+{ }_{1} \bar{\sigma}^{33}+{ }_{0} \bar{b}^{3}=0,
\end{aligned}
$$

and

$$
\begin{gathered}
{ }_{1} \bar{\sigma}^{11}{ }_{, 1}-\frac{R^{\prime \prime}}{A_{1}^{3}}\left({ }_{1} \bar{\sigma}^{31}+{ }_{1} \bar{\sigma}^{13}\right)+\frac{1}{R A_{1}}\left(R^{\prime}{ }_{1} \bar{\sigma}^{11}+{ }_{1} \bar{\sigma}^{13}\right)-\frac{R R^{\prime}}{A_{1}}{ }_{1} \bar{\sigma}^{22}+2{ }_{2} \bar{\sigma}^{13}+{ }_{1} \bar{b}^{1}=0, \\
{ }_{1} \bar{\sigma}^{21}{ }_{, 1}-\frac{R^{\prime \prime}}{A_{1}^{3}}{ }_{1} \bar{\sigma}^{23}+\frac{R^{\prime}}{R A_{1}}\left({ }_{1} \bar{\sigma}^{12}+2{ }_{1} \bar{\sigma}^{21}\right)+\frac{1}{R A_{1}}\left({ }_{1} \bar{\sigma}^{23}+{ }_{1} \bar{\sigma}^{32}\right)+2{ }_{2} \bar{\sigma}^{23}+{ }_{1} \bar{b}^{2}=0, \\
{ }_{1} \bar{\sigma}^{31}{ }_{, 1}+\frac{R^{\prime \prime}}{A_{1}^{3}}\left({ }_{1} \bar{\sigma}^{11}-{ }_{1} \bar{\sigma}^{33}\right)+\frac{1}{R A_{1}}\left(R^{\prime}{ }_{1} \bar{\sigma}^{31}+{ }_{1} \bar{\sigma}^{33}\right)-\frac{R}{A_{1}}{ }_{1} \bar{\sigma}^{22}+2{ }_{2} \bar{\sigma}^{33}+{ }_{1} \bar{b}^{3}=0 .
\end{gathered}
$$

The rotation components $\bar{\phi}^{m}\left(\xi^{1}\right)$ and the displacement components $\bar{u}_{n}\left(\xi^{1}\right)$ are approximated by polynomials of the first-degree in $\xi^{3}$ :

$$
\bar{\phi}^{m}\left(\xi^{1}, \xi^{3}\right)={ }_{0} \bar{\phi}^{m}\left(\xi^{1}\right)+{ }_{1} \bar{\phi}^{m}\left(\xi^{1}\right) \xi^{3},
$$

and

$$
\bar{u}_{n}\left(\xi^{1}, \xi^{3}\right)={ }_{0} \bar{u}_{n}\left(\xi^{1}\right)+{ }_{1} \bar{u}_{n}\left(\xi^{1}\right) \xi^{3},
$$

where ${ }_{0} \bar{\phi}^{m},{ }_{1} \bar{\phi}^{m}$ and ${ }_{0} \bar{u}_{n},{ }_{1} \bar{u}_{n}$ are, respectively, the rotation and displacement coefficients.

4.2. Stress boundary conditions and prescribed displacements. The stress boundary conditions on the top and bottom surfaces $S^{ \pm}$of the shell can be written in the form

$$
\boldsymbol{\sigma} \cdot \mathbf{n}^{ \pm}=\boldsymbol{\sigma} \cdot\left( \pm \mathbf{a}_{3}\right)= \pm \bar{\sigma}^{k 3} \mathbf{a}_{k}=\widetilde{\mathbf{p}}^{ \pm}, \quad \pm \bar{\sigma}^{k 3}=\left(\widetilde{\bar{p}}^{ \pm}\right)^{k} \quad \xi^{m} \in S^{ \pm},
$$

where the outward unit normals are denoted by

$$
\mathbf{n}^{ \pm}=\bar{n}^{ \pm} \mathbf{a}_{3}, \quad \bar{n}^{ \pm}=\left\{\begin{array}{cc}
-1 & \text { on } S^{-}, \\
1 & \text { on } S^{+}
\end{array}\right.
$$

and $\widetilde{\mathbf{p}}^{ \pm}$are prescribed surface tractions on $S^{ \pm}$. Substituting 4.8 into the stress boundary conditions (4.17) and taking into account (4.18), we obtain the following equations:

$$
\begin{aligned}
{ }_{0} \bar{\sigma}^{k 3}+{ }_{1} \bar{\sigma}^{k 3} \frac{d}{2}+{ }_{2} \bar{\sigma}^{k 3} \frac{d^{2}}{4}=\left(\tilde{\bar{p}}^{+}\right)^{k}, \\
{ }_{0} \bar{\sigma}^{k 3}+{ }_{1} \bar{\sigma}^{k 3} \frac{d}{2}-{ }_{2} \bar{\sigma}^{k 3} \frac{d^{2}}{4}=\left(\widetilde{\bar{p}}^{-}\right)^{k} .
\end{aligned}
$$

Adding and subtracting the above equations, the one-dimensional vector-valued load coefficients on $S^{ \pm}$are given by

$$
{ }_{0} \widetilde{\bar{p}}^{k}\left(\xi^{1}\right)=\frac{1}{2}\left[\left(\widetilde{\bar{p}}^{+}\right)^{k}+\left(\widetilde{\bar{p}}^{-}\right)^{k}\right]
$$




$$
{ }_{1} \overline{\bar{p}}^{k}\left(\xi^{1}\right)=\frac{1}{d}\left[\left(\tilde{\bar{p}}^{+}\right)^{k}-\left(\tilde{\bar{p}}^{-}\right)^{k}\right] .
$$

Making use of equations 4.21 -4.22, the stress boundary conditions 4.19-4.20 can be manipulated into the form

$$
\begin{aligned}
{ }_{1} \bar{\sigma}^{k 3} & =\frac{2}{d}{ }_{0} \overline{\bar{p}}^{k} \\
{ }_{2} \bar{\sigma}^{k 3} & =-\frac{4}{d^{2}}{ }_{0} \bar{\sigma}^{k 3}+\frac{2}{d}{ }_{1} \widetilde{\bar{p}}^{k},
\end{aligned}
$$

where

$$
\widetilde{\bar{p}}^{k}\left(\xi^{1}, \xi^{3}\right)={ }_{0} \widetilde{\bar{p}}^{k}+{ }_{1} \widetilde{\bar{p}}^{k} \xi^{3},
$$

and

$$
\widetilde{\bar{p}}^{k}\left(\xi^{1}, \xi^{3}= \pm d / 2\right)=\left(\widetilde{\bar{p}}^{ \pm}\right)^{k}={ }_{0} \widetilde{\bar{p}}^{k} \pm \frac{d}{2}{ }_{1} \widetilde{\bar{p}}^{k} .
$$

The outward unit normals of the lateral surfaces $S^{0}$ and $S^{L}$ can be given by

$$
\mathbf{n}^{\times}=\bar{n}^{\times} \mathbf{a}_{1}, \quad \bar{n}^{\times}=\left\{\begin{array}{cc}
-1 & \text { on } S^{0}, \\
1 & \text { on } S^{L} .
\end{array}\right.
$$

Let $\widetilde{\mathbf{p}}^{\times}$be the prescribed surface traction on the lateral surface $S^{L}$ with outward unit normal $\mathbf{n}^{\times}$defined by equation 4.27). The stress boundary conditions on $S^{L}$ can be written in the form

$$
\boldsymbol{\sigma} \cdot \mathbf{n}^{\times}=\boldsymbol{\sigma} \cdot \mathbf{a}^{1}=\bar{\sigma}^{k 1} \mathbf{a}_{k}=\widetilde{\mathbf{p}}^{\times}, \quad \bar{\sigma}^{k 1}=\left(\widetilde{\bar{p}}^{\times}\right)^{k} \quad \xi^{m} \in S^{L} .
$$

Expanding the prescribed lateral surface tractions $\left(\tilde{\bar{p}}^{\times}\right)^{k}$ into a truncated power series with respect to $\xi^{3}$ and substituting 4.7 into 4.28 , we obtain the following stress boundary conditions on the lateral surface $S^{L}$ :

$$
\begin{array}{ll}
\left.{ }_{0} \bar{\sigma}^{k 1}\right|_{x^{1}=L}={ }_{0}\left(\widetilde{\bar{p}}^{\times}\right)^{k} & \xi^{m} \in S^{L}, \\
\left.{ }_{1} \bar{\sigma}^{k 1}\right|_{x^{1}=L}={ }_{1}\left(\widetilde{\bar{p}}^{\times}\right)^{k} & \xi^{m} \in S^{L},
\end{array}
$$

where ${ }_{0}\left(\tilde{\bar{p}}^{\times}\right)^{k}$ and ${ }_{1}\left(\tilde{\bar{p}}^{\times}\right)^{k}$ are the prescribed one-dimensional vector-valued load coefficients on the lateral surface $S^{L}$.

Considering expression (4.16), the displacement boundary conditions on the lateral surface $S^{0}$ with outward unit normal $\mathbf{n}^{\times}$can be given by

$$
\bar{u}_{k}={ }_{0} \bar{u}_{k}+{ }_{1} \bar{u}_{k} \xi^{3}=\widetilde{\bar{u}}_{k}={ }_{0} \widetilde{\bar{u}}_{k}+{ }_{1} \widetilde{\bar{u}}_{k} \xi^{3} \quad \xi^{m} \in S^{0},
$$

or

$$
\begin{array}{ll}
\left.{ }_{0} \bar{u}_{k}\right|_{\xi^{1}=0}={ }_{0} \widetilde{\bar{u}}_{k} \quad & \xi^{m} \in S^{0}, \\
\left.{ }_{1} \bar{u}_{k}\right|_{\xi^{1}=0}={ }_{1} \widetilde{\bar{u}}_{k} \quad & \xi^{m} \in S^{0},
\end{array}
$$

where ${ }_{0} \widetilde{\bar{u}}_{k},{ }_{1} \widetilde{\bar{u}}_{k}$ are prescribed displacement coefficients on the lateral surface $S^{0}$. 
4.3. Euler-Lagrange equations and natural boundary conditions. Assuming linearly elastic, homogeneous and isotropic materials, the strain tensor $\bar{\varepsilon}_{k l}$ for thin shells can be expressed by the inverse stress-strain relation (Hooke's law)

$$
\bar{\varepsilon}_{k l}\left(\bar{\sigma}^{r s}\right)=\frac{1}{4 G}\left[\left(\bar{\sigma}^{r s}+\bar{\sigma}^{s r}\right) a_{r k} a_{s l}-\frac{2 \nu}{1+\nu} \bar{\sigma}^{p q} a_{p q} a_{k l}\right]
$$

where $G$ is the shear modulus of elasticity and $\nu$ is the Poisson ratio. Making use of equation 2.16, the above constitutive equations can be written in the following form:

$$
\begin{aligned}
& \bar{\varepsilon}_{11}=\frac{1}{E}\left[\bar{\sigma}^{11}-\nu\left(R^{2} \bar{\sigma}^{22}+\bar{\sigma}^{33}\right)\right], \\
& \bar{\varepsilon}_{22}=\frac{R^{2}}{E}\left[R^{2} \bar{\sigma}^{22}-\nu\left(\bar{\sigma}^{11}+\bar{\sigma}^{33}\right)\right], \\
& \bar{\varepsilon}_{33}=\frac{1}{E}\left[\bar{\sigma}^{33}-\nu\left(\bar{\sigma}^{11}+R^{2} \bar{\sigma}^{22}\right)\right], \\
& \bar{\varepsilon}_{31}=\frac{1}{4 G}\left(\bar{\sigma}^{13}+\bar{\sigma}^{31}\right)=\bar{\varepsilon}_{13} \\
& \bar{\varepsilon}_{21}=\frac{R^{2}}{4 G}\left(\bar{\sigma}^{12}+\bar{\sigma}^{21}\right)=\bar{\varepsilon}_{12} \\
& \bar{\varepsilon}_{32}=\frac{R^{2}}{4 G}\left(\bar{\sigma}^{23}+\bar{\sigma}^{32}\right)=\bar{\varepsilon}_{23},
\end{aligned}
$$

where $E=2 G(1+\nu)$ is the elasticity modulus. In the case of axisymmetric shells, the outward unit normal to the lateral surface $S_{u} \equiv S^{0}$ is defined by 4.27). In view of this, the Hellinger-Reissner functional (1.6) for thin shells can be written in the form

$$
\begin{aligned}
H R_{d}\left(\bar{\sigma}^{r s}, \bar{\phi}^{m}, \bar{u}_{n}\right) & =-\frac{1}{2} \int_{-\frac{d}{2}\left(S_{0}\right)}^{+\frac{d}{2}} \bar{\sigma}^{k l} \bar{\varepsilon}_{k l}\left(\bar{\sigma}^{r s}\right) \mathrm{d} S_{0} \mathrm{~d} \xi^{3}-\int_{-\frac{d}{2}}^{+\frac{d}{2}} \int_{0}^{2 \pi} \widetilde{\bar{u}}_{k} \bar{\sigma}^{k 1} R_{0} \mathrm{~d} \xi^{2} \mathrm{~d} \xi^{3}+ \\
& +\int_{-\frac{d}{2}\left(S_{0}\right)}^{+\frac{d}{2}} \bar{\sigma}^{k l} \bar{\epsilon}_{k l m} \bar{\phi}^{m} \mathrm{~d} S_{0} \mathrm{~d} \xi^{3}-\int_{-\frac{d}{2}\left(S_{0}\right)}^{+\frac{d}{2}} \int_{\bar{u}_{n}}\left(\bar{\sigma}_{\mid l}^{n l}+\bar{b}^{n}\right) \mathrm{d} S_{0} \mathrm{~d} \xi^{3}
\end{aligned}
$$

Since the axisymmetrical deformation means that tensor variables are independent of the coordinate $\xi^{2}$, the integration with respect to $\xi^{2}$ can easily be carried out. Thus the first variation of (4.41) with respect to stresses $\bar{\sigma}^{k l}$, rotations $\bar{\phi}^{m}$ and displacements $\bar{u}_{n}$ yields the Hellinger-Reissner variational principle in the following form

$$
\begin{aligned}
& \frac{1}{2 \pi} \delta H R_{d}\left(\bar{\sigma}^{r s}, \bar{\phi}^{m}, \bar{u}_{n}, \delta \bar{\sigma}^{r s}, \delta \bar{\phi}^{m}, \delta \bar{u}_{n}\right)= \\
& \quad-\int_{-\frac{d}{2}}^{+\frac{d}{2}} \int_{0}^{s_{L}} \delta \bar{\sigma}^{k l} \bar{\varepsilon}_{k l} R \mathrm{~d} \xi^{1} \mathrm{~d} \xi^{3}-\int_{-\frac{d}{2}}^{+\frac{d}{2}} \overline{\bar{u}}_{k} \delta \bar{\sigma}^{k 1} R_{0} \mathrm{~d} \xi^{3}+\int_{-\frac{d}{2}}^{+\frac{d}{2}} \int_{0}^{s_{L}} \delta \bar{\sigma}^{k l} \bar{\epsilon}_{k l m} \bar{\phi}^{m} R \mathrm{~d} \xi^{1} \mathrm{~d} \xi^{3}+
\end{aligned}
$$


$+\int_{-\frac{d}{2}}^{+\frac{d}{2}} \int_{0}^{s_{L}} \bar{\sigma}^{k l} \bar{\epsilon}_{k l m} \delta \bar{\phi}^{m} R \mathrm{~d} \xi^{1} \mathrm{~d} \xi^{3}-\int_{-\frac{d}{2}}^{+\frac{d}{2}} \int_{0}^{s_{L}} \bar{u}_{n} \delta \bar{\sigma}_{\mid l}^{n l} R \mathrm{~d} \xi^{1} \mathrm{~d} \xi^{3}-\int_{-\frac{d}{2}}^{+\frac{d}{2}} \int_{0}^{s_{L}} \delta \bar{u}_{n}\left(\bar{\sigma}_{\mid l}^{n l}+\bar{b}^{n}\right) R \mathrm{~d} \xi^{1} \mathrm{~d} \xi^{3}=0$,

where

$$
s_{L}=\int_{\hat{x}^{1}=0}^{L} A_{1}\left(\hat{x}^{1}\right) \mathrm{d} \hat{x}^{1}
$$

is the arc-length measured along the meridian curve.

4.3.1. The first variation of the complementary strain energy. Making use of the expanded stress components 4.7 - 4.8), the first variation of the complementary strain energy of the shell can be written as

$$
\begin{aligned}
\frac{1}{2 \pi} \int_{(V)} \delta W_{c}\left(\bar{\sigma}^{r s}\right) \mathrm{d} V=\int_{-\frac{d}{2}}^{+\frac{d}{2}} \int_{0}^{s_{L}} \delta \bar{\sigma}^{k l} \bar{\varepsilon}_{k l} R \mathrm{~d} \xi^{1} \mathrm{~d} \xi^{3}= \\
=\int_{-\frac{d}{2}}^{+\frac{d}{2}} \int_{0}^{s_{L}}\left[\delta_{0} \bar{\sigma}^{k l} \bar{\varepsilon}_{k l}+\delta_{1} \bar{\sigma}^{k l} \bar{\varepsilon}_{k l} \xi^{3}+\delta_{2} \bar{\sigma}^{k 3} \bar{\varepsilon}_{k 3}\left(\xi^{3}\right)^{2}\right] R \mathrm{~d} \xi^{1} \mathrm{~d} \xi^{3} .
\end{aligned}
$$

Introducing the strain resultants

$$
\overline{\mathrm{E}}_{k \ell}^{(0)}:=\int_{-\frac{d}{2}}^{+\frac{d}{2}} \bar{\varepsilon}_{k \ell} \mathrm{d} \xi^{3}, \quad \overline{\mathrm{E}}_{k \ell}^{(1)}:=\int_{-\frac{d}{2}}^{+\frac{d}{2}} \bar{\varepsilon}_{k \ell} \xi^{3} \mathrm{~d} \xi^{3}, \quad \overline{\mathrm{E}}_{k 3}^{(2)}:=\int_{-\frac{d}{2}}^{+\frac{d}{2}} \bar{\varepsilon}_{k 3}\left(\xi^{3}\right)^{2} \mathrm{~d} \xi^{3}
$$

and inserting them into equation (4.44 the following expression is obtained:

$$
\begin{aligned}
\frac{1}{2 \pi} \int_{(V)} \delta W_{c}\left(\bar{\sigma}^{r s}\right) \mathrm{d} V & =\int_{-\frac{d}{2}}^{+\frac{d}{2}} \int_{0}^{s_{L}} \delta \bar{\sigma}^{k l} \bar{\varepsilon}_{k l} R \mathrm{~d} \xi^{1} \mathrm{~d} \xi^{3}= \\
& =\int_{0}^{s_{L}}\left[\delta_{0} \bar{\sigma}^{k l} \overline{\mathrm{E}}_{k \ell}^{(0)}+\delta_{1} \bar{\sigma}^{k l} \overline{\mathrm{E}}_{k \ell}^{(1)}+\delta_{2} \bar{\sigma}^{k 3} \overline{\mathrm{E}}_{k 3}^{(2)}\right] R \mathrm{~d} \xi^{1}
\end{aligned}
$$

4.3.2. The first variation of the boundary integral term. Making use of the expanded stress components (4.7), the first variation of the boundary integral in equation (4.41) in terms of the variations of the one-dimensional stress coefficients can be written as

$$
-\int_{-\frac{d}{2}}^{+\frac{d}{2}} \widetilde{\bar{u}}_{k} \delta \bar{\sigma}^{k 1} R_{0} \mathrm{~d} \xi^{3}=-\int_{-\frac{d}{2}}^{+\frac{d}{2}}\left[\widetilde{\bar{u}}_{k} \delta_{0} \bar{\sigma}^{k 1}+\widetilde{\bar{u}}_{k} \delta_{1} \bar{\sigma}^{k 1} \xi^{3}\right] R_{0} \mathrm{~d} \xi^{3} .
$$


Introducing the prescribed displacement resultants

$$
\widetilde{\overline{\mathrm{U}}}_{k}^{(0)}:=\int_{-\frac{d}{2}}^{+\frac{d}{2}} \widetilde{\bar{u}}_{k} \mathrm{~d} \xi^{3}, \quad \widetilde{\overline{\mathrm{U}}}_{k}^{(1)}:=\int_{-\frac{d}{2}}^{+\frac{d}{2}} \widetilde{\bar{u}}_{k} \xi^{3} \mathrm{~d} \xi^{3}
$$

and substituting these into equation (4.47), the first variation of the boundary integral term takes the form

$$
-\int_{-\frac{d}{2}}^{+\frac{d}{2}} \widetilde{\bar{u}}_{k} \delta \bar{\sigma}^{k 1} R_{0} \mathrm{~d} \xi^{3}=-R_{0} \widetilde{\overline{\mathrm{U}}}_{k}^{(0)} \delta_{0} \bar{\sigma}^{k 1}-R_{0} \widetilde{\overline{\mathrm{U}}}_{k}^{(1)} \delta_{1} \bar{\sigma}^{k 1} .
$$

4.3.3. The first variation of the Lagrangian multiplier terms. Making use of the expanded stress components (4.7)-4.8) and rotation components 4.15), the first variations of the Lagrangian multiplier term

$$
\int_{-\frac{d}{2}\left(S_{0}\right)}^{+\frac{d}{2}} \int^{k l} \bar{\epsilon}_{k l m} \bar{\phi}^{m} \mathrm{~d} S_{0} \mathrm{~d} \xi^{3}
$$

in functional 4.41 with respect to the stresses $\bar{\sigma}^{k l}$ and the rotations $\bar{\phi}^{m}$ are, respectively,

$$
\begin{aligned}
& \int_{-\frac{d}{2}}^{+\frac{d}{2}} \int_{0}^{s_{L}} \delta \bar{\sigma}^{k l} \bar{\epsilon}_{k l m} \bar{\phi}^{m} R \mathrm{~d} \xi^{1} \mathrm{~d} \xi^{3}= \\
& =\int_{-\frac{d}{2}}^{+\frac{d}{2}} \int_{0}^{s_{L}}\left[\delta_{0} \bar{\sigma}^{k l} \bar{\epsilon}_{k l m} \bar{\phi}^{m}+\delta_{1} \bar{\sigma}^{k l} \bar{\epsilon}_{k l m} \bar{\phi}^{m} \xi^{3}+\delta_{2} \bar{\sigma}^{k 3} \bar{\epsilon}_{k 3 m} \bar{\phi}^{m}\left(\xi^{3}\right)^{2}\right] R \mathrm{~d} \xi^{1} \mathrm{~d} \xi^{3}
\end{aligned}
$$

and

$$
\int_{-\frac{d}{2}}^{+\frac{d}{2}} \int_{0}^{s_{L}} \bar{\sigma}^{k l_{\bar{\epsilon}}} \bar{\epsilon}_{k l m} \delta \bar{\phi}^{m} R \mathrm{~d} \xi^{1} \mathrm{~d} \xi^{3}=\int_{-\frac{d}{2}}^{+\frac{d}{2}} \int_{0}^{s_{L}}\left[\bar{\sigma}^{k l} \bar{\epsilon}_{k l m} \delta_{0} \bar{\phi}^{m}+\bar{\sigma}^{k l} \bar{\epsilon}_{k l m} \delta_{1} \bar{\phi}^{m} \xi^{3}\right] R \mathrm{~d} \xi^{1} \mathrm{~d} \xi^{3} .
$$

Introducing the rotation resultants

$$
\bar{\Phi}_{k l}^{(0)}:=-\int_{-\frac{d}{2}}^{+\frac{d}{2}} \bar{\epsilon}_{k l m} \bar{\phi}^{m} \mathrm{~d} \xi^{3}, \bar{\Phi}_{k l}^{(1)}:=-\int_{-\frac{d}{2}}^{+\frac{d}{2}} \bar{\epsilon}_{k l m} \bar{\phi}^{m} \xi^{3} \mathrm{~d} \xi^{3}, \bar{\Phi}_{k 3}^{(2)}:=-\int_{-\frac{d}{2}}^{+\frac{d}{2}} \bar{\epsilon}_{k 3 m} \bar{\phi}^{m}\left(\xi^{3}\right)^{2} \mathrm{~d} \xi^{3}
$$


and substituting them into equation 4.51, expression 4.51) can be written in the following form:

$$
\int_{-\frac{d}{2}}^{+\frac{d}{2}} \int_{0}^{s_{L}} \delta \bar{\sigma}^{k l} \bar{\epsilon}_{k l m} \bar{\phi}^{m} R \mathrm{~d} \xi^{1} \mathrm{~d} \xi^{3}=-\int_{0}^{s_{L}}\left[\delta_{0} \bar{\sigma}^{k l} \bar{\Phi}_{k l}^{(0)}+\delta_{1} \bar{\sigma}^{k l} \bar{\Phi}_{k l}^{(1)}+\delta_{0} \bar{\sigma}^{k 3} \bar{\Phi}_{k 3}^{(2)}\right] R \mathrm{~d} \xi^{1} .
$$

Let us investigate the integral 4.52 appearing in 4.42 . Substituting the expanded stress components (4.7)-4.8 into (4.52) and carrying out integrations with respect to $\xi^{3}$, the equation 4.52 can be written as

$$
\begin{aligned}
& \int_{-\frac{d}{2}}^{+\frac{d}{2}} \int_{0}^{s_{L}} \bar{\sigma}^{k l} \bar{\epsilon}_{k l m} \delta \bar{\phi}^{m} R \mathrm{~d} \xi^{1} \mathrm{~d} \xi^{3}= \\
& \int_{0}^{s_{L}}\left\{d\left[\left({ }_{0} \bar{\sigma}^{23}-{ }_{0} \bar{\sigma}^{32}\right) \delta_{0} \bar{\phi}^{1}+\left({ }_{0} \bar{\sigma}^{31}-{ }_{0} \bar{\sigma}^{13}\right) \delta_{0} \bar{\phi}^{2}+\left({ }_{0} \bar{\sigma}^{12}-{ }_{0} \bar{\sigma}^{21}\right) \delta_{0} \bar{\phi}^{3}\right]+\right. \\
& +\frac{d^{3}}{12}\left[\left({ }_{1} \bar{\sigma}^{23}-{ }_{1} \bar{\sigma}^{32}\right) \delta_{1} \bar{\phi}^{1}+\left({ }_{1} \bar{\sigma}^{31}-{ }_{1} \bar{\sigma}^{13}\right) \delta_{1} \bar{\phi}^{2}+\left({ }_{1} \bar{\sigma}^{12}-{ }_{1} \bar{\sigma}^{21}\right) \delta_{1} \bar{\phi}^{3}+\right. \\
& \left.\left.+{ }_{2} \bar{\sigma}^{23} \delta_{0} \bar{\phi}^{1}-{ }_{2} \bar{\sigma}^{13} \delta_{0} \bar{\phi}^{2}\right]\right\} R^{2} \mathrm{~d} \xi^{1}
\end{aligned}
$$

According to 4.42 , the coefficients of the rotation components $\delta_{0} \bar{\phi}^{m}, \delta_{1} \bar{\phi}^{m}$ in the above expression should be equal to zero:

$$
\begin{aligned}
& \delta_{0} \bar{\phi}^{1}: \quad{ }_{0} \bar{\sigma}^{32}={ }_{0} \bar{\sigma}^{23}+\frac{d^{2}}{12}{ }_{2} \bar{\sigma}^{23}, \\
& \delta_{0} \bar{\phi}^{2}: \quad{ }_{0} \bar{\sigma}^{31}={ }_{0} \bar{\sigma}^{13}+\frac{d^{2}}{12}{ }_{2} \bar{\sigma}^{13}, \\
& \delta_{0} \bar{\phi}^{3}: \quad{ }_{0} \bar{\sigma}^{12}={ }_{0} \bar{\sigma}^{21}, \\
& \delta_{1} \bar{\phi}^{1}: \quad{ }_{1} \bar{\sigma}^{32}={ }_{1} \bar{\sigma}^{23}, \\
& \delta_{1} \bar{\phi}^{2}: \quad{ }_{1} \bar{\sigma}^{31}={ }_{1} \bar{\sigma}^{13}, \\
& \delta_{1} \bar{\phi}^{3}: \quad{ }_{1} \bar{\sigma}^{12}={ }_{1} \bar{\sigma}^{21} \text {. }
\end{aligned}
$$

It can easily be seen that equations 4.56-4.57) and $4.59-4.60$ are equivalent to the symmetry of the transverse shear stresses satisfied in integral average sense. The advantage of equations 4.56-4.61 is that ${ }_{0} \bar{\sigma}^{32},{ }_{0} \bar{\sigma}^{31},{ }_{0} \bar{\sigma}^{12},{ }_{1} \bar{\sigma}^{32},{ }_{1} \bar{\sigma}^{31}$ and ${ }_{1} \bar{\sigma}^{12}$ are expressed in terms of the stress coefficients ${ }_{0} \bar{\sigma}^{23}$ and ${ }_{2} \bar{\sigma}^{23},{ }_{0} \bar{\sigma}^{13}$ and ${ }_{2} \bar{\sigma}^{13},{ }_{0} \bar{\sigma}^{21}$, ${ }_{1} \bar{\sigma}^{23},{ }_{1} \bar{\sigma}^{13}$ and ${ }_{1} \bar{\sigma}^{21}$, respectively. Thus, not only the number of rotation variables but also the number of stress variables could further be reduced. After substituting the expanded displacement components 4.16 into equation 4.42 and carrying out the integration with respect to $\xi^{3}$, the coefficients of the displacement components 
$\delta_{0} \bar{u}_{k}, \delta_{1} \bar{u}_{k}$ should be equal to zero:

$$
\begin{aligned}
& { }_{0} \bar{\sigma}^{11}{ }_{, 1}-\frac{R^{\prime \prime}}{A_{1}^{3}}\left({ }_{0} \bar{\sigma}^{31}+{ }_{0} \bar{\sigma}^{13}\right)+\frac{1}{R A_{1}}\left(R^{\prime}{ }_{0} \bar{\sigma}^{11}+{ }_{0} \bar{\sigma}^{13}\right)-\frac{R R^{\prime}}{A_{1}}{ }_{0} \bar{\sigma}^{22}+{ }_{1} \bar{\sigma}^{13}+{ }_{0} \bar{b}^{1}=0, \\
& { }_{0} \bar{\sigma}^{21}{ }_{, 1}-\frac{R^{\prime \prime}}{A_{1}^{3}}{ }_{0} \bar{\sigma}^{23}+\frac{R^{\prime}}{R A_{1}}\left({ }_{0} \bar{\sigma}^{12}+2{ }_{0} \bar{\sigma}^{21}\right)+\frac{1}{R A_{1}}\left({ }_{0} \bar{\sigma}^{23}+{ }_{0} \bar{\sigma}^{32}\right)+{ }_{1} \bar{\sigma}^{23}+{ }_{0} \bar{b}^{2}=0, \\
& { }_{0} \bar{\sigma}^{31}{ }_{, 1}+\frac{R^{\prime \prime}}{A_{1}^{3}}\left({ }_{0} \bar{\sigma}^{11}-{ }_{0} \bar{\sigma}^{33}\right)+\frac{1}{R A_{1}}\left(R^{\prime}{ }_{0} \bar{\sigma}^{31}+{ }_{0} \bar{\sigma}^{33}\right)-\frac{R}{A_{1}}{ }_{0} \bar{\sigma}^{22}+{ }_{1} \bar{\sigma}^{33}+{ }_{0} \bar{b}^{3}=0,
\end{aligned}
$$

and

$$
\begin{gathered}
{ }_{1} \bar{\sigma}^{11}{ }_{1}-\frac{R^{\prime \prime}}{A_{1}^{3}}\left({ }_{1} \bar{\sigma}^{31}+{ }_{1} \bar{\sigma}^{13}\right)+\frac{1}{R A_{1}}\left(R^{\prime}{ }_{1} \bar{\sigma}^{11}+{ }_{1} \bar{\sigma}^{13}\right)-\frac{R R^{\prime}}{A_{1}}{ }_{1} \bar{\sigma}^{22}+2{ }_{2} \bar{\sigma}^{13}+{ }_{1} \bar{b}^{1}=0, \\
{ }_{1} \bar{\sigma}^{21}{ }_{1}-\frac{R^{\prime \prime}}{A_{1}^{3}}{ }_{1} \bar{\sigma}^{23}+\frac{R^{\prime}}{R A_{1}}\left({ }_{1} \bar{\sigma}^{12}+2{ }_{1} \bar{\sigma}^{21}\right)+\frac{1}{R A_{1}}\left({ }_{1} \bar{\sigma}^{23}+{ }_{1} \bar{\sigma}^{32}\right)+2{ }_{2} \bar{\sigma}^{23}+{ }_{1} \bar{b}^{2}=0, \\
{ }_{1} \bar{\sigma}^{31}{ }_{, 1}+\frac{R^{\prime \prime}}{A_{1}^{3}}\left({ }_{1} \bar{\sigma}^{11}-{ }_{1} \bar{\sigma}^{33}\right)+\frac{1}{R A_{1}}\left(R^{\prime}{ }_{1} \bar{\sigma}^{31}+{ }_{1} \bar{\sigma}^{33}\right)-\frac{R}{A_{1}}{ }_{1} \bar{\sigma}^{22}+2{ }_{2} \bar{\sigma}^{33}+{ }_{1} \bar{b}^{3}=0 .
\end{gathered}
$$

Thus we have obtained the one-dimensional equilibrium equations 4.9 - 4.14 for the one-dimensional stress coefficients.

Applying a similar procedure to the Lagrangian multiplier term

$$
\int_{-\frac{d}{2}}^{+\frac{d}{2}} \int_{0}^{s_{L}} \bar{u}_{n} \delta \bar{\sigma}_{\mid l}^{n l} R \mathrm{~d} \xi^{1} \mathrm{~d} \xi^{3}
$$

and taking into account the integration rule

$$
\begin{aligned}
& \int_{0}^{s_{L}}{ }_{i} \bar{u}_{n} \delta_{i} \bar{\sigma}_{, 1}^{n 1} R \mathrm{~d} \xi^{1}=\left[{ }_{i} \bar{u}_{n} \delta_{i} \bar{\sigma}^{n 1} R\right]_{\xi^{1}=0}^{\xi^{1}=s_{L}}- \\
& -\int_{0}^{s_{L}} \bar{u}_{n, 1} \delta_{i} \bar{\sigma}^{n 1} R \mathrm{~d} \xi^{1}-\int_{0}^{s_{L}} \bar{u}_{n} \delta_{i} \bar{\sigma}^{n 1} \frac{R^{\prime}}{A_{1}} \mathrm{~d} \xi^{1}, \quad i=0,1,
\end{aligned}
$$

as well as expressions 4.46, 4.49 and 4.54, the coefficients of $\delta_{0} \bar{\sigma}^{k l}, \delta_{1} \bar{\sigma}^{k l}$ and $\delta_{2} \bar{\sigma}^{k 3}$ should be equal to zero, yielding the kinematic equations

$$
\begin{array}{ll}
\delta_{0} \bar{\sigma}^{11}: & d_{0} \bar{u}_{1,1}-d \frac{R^{\prime \prime}}{A_{1}^{3}}{ }_{0} \bar{u}_{3}-\overline{\mathrm{E}}_{11}^{(0)}=0, \\
\delta_{0} \bar{\sigma}^{12}: & -d \frac{R^{\prime}}{A_{1}}{ }_{0} \bar{u}_{2}-R \bar{\Phi}_{12}^{(0)}-R \overline{\mathrm{E}}_{12}^{(0)}=0, \\
\delta_{0} \bar{\sigma}^{13}: & d\left(\frac{R R^{\prime \prime}}{A_{1}^{3}}-\frac{1}{A_{1}}\right){ }_{0} \bar{u}_{1}-R \bar{\Phi}_{13}^{(0)}-R \overline{\mathrm{E}}_{13}^{(0)}=0,
\end{array}
$$




$$
\begin{array}{ll}
\delta_{0} \bar{\sigma}^{21}: & d R_{0} \bar{u}_{2,1}-d \frac{R^{\prime}}{A_{1}}{ }_{0} \bar{u}_{2}-R \bar{\Phi}_{21}^{(0)}-R \overline{\mathrm{E}}_{21}^{(0)}=0, \\
\delta_{0} \bar{\sigma}^{22}: & d \frac{R R^{\prime}}{A_{1}}{ }_{0} \bar{u}_{1}+d \frac{R}{A_{1}}{ }_{0} \bar{u}_{3}-\overline{\mathrm{E}}_{22}^{(0)}=0, \\
\delta_{0} \bar{\sigma}^{23}: & d\left(\frac{R R^{\prime \prime}}{A_{1}^{3}}-\frac{1}{A_{1}}\right){ }_{0} \bar{u}_{2}-R \bar{\Phi}_{23}^{(0)}-R \overline{\mathrm{E}}_{23}^{(0)}=0, \\
\delta_{0} \bar{\sigma}^{31}: & d_{0} \bar{u}_{3,1}+d \frac{R^{\prime \prime}}{A_{1}^{3}}{ }_{0} \bar{u}_{1}-\bar{\Phi}_{31}^{(0)}-\overline{\mathrm{E}}_{31}^{(0)}=0, \\
\delta_{0} \bar{\sigma}^{32}: & -\frac{d}{A_{1}}{ }_{0} \bar{u}_{2}-R \bar{\Phi}_{32}^{(0)}-R \overline{\mathrm{E}}_{32}^{(0)}=0, \\
\delta_{0} \bar{\sigma}^{33}: & d\left(\frac{R R^{\prime \prime}}{A_{1}^{3}}-\frac{1}{A_{1}}\right){ }_{0} \bar{u}_{3}-R \overline{\mathrm{E}}_{33}^{(0)}=0,
\end{array}
$$

and

$$
\begin{aligned}
& \delta_{1} \bar{\sigma}^{11}: \quad \frac{d^{3}}{12}\left({ }_{1} \bar{u}_{1,1}+\frac{R^{\prime \prime}}{A_{1}^{3}}{ }_{1} \bar{u}_{3}\right)-\overline{\mathrm{E}}_{11}^{(1)}=0, \\
& \delta_{1} \bar{\sigma}^{12}: \quad-\frac{d^{3}}{12} \frac{R^{\prime}}{A_{1}}{ }_{1} \bar{u}_{2}-R \bar{\Phi}_{12}^{(1)}-R \overline{\mathrm{E}}_{12}^{(1)}=0, \\
& \delta_{1} \bar{\sigma}^{13}: \quad \frac{d^{3}}{12}\left(\frac{R R^{\prime \prime}}{A_{1}^{3}}-\frac{1}{A_{1}}\right){ }_{1} \bar{u}_{1}-d R_{0} \bar{u}_{1}-R \bar{\Phi}_{13}^{(1)}-R \overline{\mathrm{E}}_{13}^{(1)}=0, \\
& \delta_{1} \bar{\sigma}^{21}: \quad \frac{d^{3}}{12}\left(R_{1} \bar{u}_{2,1}-\frac{R^{\prime}}{A_{1}}{ }_{1} \bar{u}_{2}\right)-R \bar{\Phi}_{21}^{(1)}-R \overline{\mathrm{E}}_{21}^{(1)}=0, \\
& \delta_{1} \bar{\sigma}^{22}: \quad \frac{d^{3}}{12}\left(\frac{R R^{\prime}}{A_{1}}{ }_{1} \bar{u}_{1}+\frac{R}{A_{1}}{ }_{1} \bar{u}_{3}\right)-\overline{\mathrm{E}}_{22}^{(1)}=0, \\
& \delta_{1} \bar{\sigma}^{23}: \quad \frac{d^{3}}{12}\left(\frac{R R^{\prime \prime}}{A_{1}^{3}}-\frac{1}{A_{1}}\right){ }_{1} \bar{u}_{2}-d R_{1} \bar{u}_{2}-R \bar{\Phi}_{23}^{(1)}-R \overline{\mathrm{E}}_{23}^{(1)}=0, \\
& \delta_{1} \bar{\sigma}^{31}: \quad \frac{d^{3}}{12}\left({ }_{1} \bar{u}_{3,1}+\frac{R^{\prime \prime}}{A_{1}^{3}}{ }_{1} \bar{u}_{1}\right)-\bar{\Phi}_{31}^{(1)}-\overline{\mathrm{E}}_{31}^{(1)}=0, \\
& \delta_{1} \bar{\sigma}^{32}: \quad-\frac{d^{3}}{12 A_{1}}{ }_{1} \bar{u}_{2}-R \bar{\Phi}_{32}^{(1)}-R \overline{\mathrm{E}}_{32}^{(1)}=0, \\
& \delta_{1} \bar{\sigma}^{33}: \quad \frac{d^{3}}{12}\left(\frac{R R^{\prime \prime}}{A_{1}^{3}}-\frac{1}{A_{1}}\right){ }_{1} \bar{u}_{3}-d R_{0} \bar{u}_{3}-R \overline{\mathrm{E}}_{33}^{(1)}=0, \\
& \delta_{2} \bar{\sigma}^{13}: \quad-\frac{d^{3}}{6}{ }_{1} \bar{u}_{1}-\bar{\Phi}_{13}^{(2)}-\overline{\mathrm{E}}_{13}^{(2)}=0, \\
& \delta_{2} \bar{\sigma}^{23}: \quad-\frac{d^{3}}{6}{ }_{1} \bar{u}_{2}-\bar{\Phi}_{23}^{(2)}-\overline{\mathrm{E}}_{23}^{(2)}=0, \\
& \delta_{2} \bar{\sigma}^{33}: \quad-\frac{d^{3}}{6}{ }_{1} \bar{u}_{3}-\overline{\mathrm{E}}_{33}^{(2)}=0,
\end{aligned}
$$


and the natural boundary conditions

$$
\begin{array}{ll}
\delta_{0} \bar{\sigma}^{n 1}: & -\left.d_{0} \bar{u}_{n}\right|_{\xi^{1}=0}-\widetilde{\overline{\mathrm{U}}}_{n}^{(0)}=0, \\
\delta_{1} \bar{\sigma}^{n 1}: & -\left.\frac{d^{3}}{12}{ }_{1} \bar{u}_{n}\right|_{\xi^{1}=0}-\widetilde{\overline{\mathrm{U}}}_{n}^{(1)}=0 .
\end{array}
$$

The Euler-Lagrange equations of Hellinger-Reissner's variational principle with functional 4.41) are the rotational and translational equilibrium equations, 4.56 - 4.61) and 4.62 - 4.67), respectively, the kinematic equations 4.70-4.90 and the displacement boundary conditions 4.91-4.92.

\section{Conclusions}

A new dimensionally reduced shell model in terms of displacements, rotations and non-symmetric stresses has been derived for axisymmetric shells applying the threedimensional dual-mixed variational principle of Hellinger-Reissner. The displacement and rotation fields appearing in this principle are considered as Lagrangian multipliers that enforce the translational and the rotational equilibrium equations into the functional of the total complementary energy. A priori satisfaction of the stress boundary conditions is required, boundary conditions for the rotations cannot be prescribed.

After describing the geometry of the shell of revolution, the main steps of the dimensional reduction in terms of displacements, rotations and non-symmetric stresses are presented. Applying a special form of the Hellinger-Reissner functional derived for shells of revolution and introducing the notion of strain, rotation and prescribed displacement resultants, the Euler-Lagrange equations and the natural boundary conditions of the dimensionally reduced shell model are derived, assuming axisymmetrical loads. These equations, written in terms of one-dimensional variables defined on the middle surface of the shell, consist of the special forms of the translational and rotational equilibrium equations, the kinematic equations and the displacement boundary conditions. The Euler-Lagrange equations obtained may be used for analytical investigations of shells of revolution with special geometry, especially when closed-form solutions in terms of the stress field can be constructed.

The most important features of the shell model derived and presented in this paper can be summarized as follows:

- Classical kinematical hypotheses regarding the deformation of the normal to the shell middle surface are not applied.

- The transverse normal stress distribution is parabolic across the thickness of the shell. This allows for the deformation of the shell in the thickness direction.

- Unmodified three-dimensional constitutive equations are applied and, at the same time, the inverse stress-strain relations for linearly elastic isotropic materials do not contain the $1 /(1-2 \nu)$ term. This fact has importance from the point of view of an incompressibility locking-free finite element formulation, when the Poisson ratio $\nu$ is close to the incompressible limit of 0.5 . 
The finite element formulation and the development of new $h p$-version dual-mixed finite element models based on the shell theory presented in this paper are the next step of the research work in this direction.

Acknowledgements. This work was supported by the Hungarian Scientific Research Fund under Grant No. OTKA T49427 and by the European Union and the State of Hungary, co-financed by the European Social Fund in the framework of TÁMOP-4.2.4.A/2-11/1-20120001 ,National Excellence Program”.

\section{REFERENCES}

1. BÉDA, Gy., KozÁK, I.: Mechanics of elastic bodies, Müszaki Könyvkiadó, Budapest, 1987 (in Hungarian).

2. BÉdA, Gy., KozÁK, I. and Verhás, J.: Continuum Mechanics, Akadémiai Kiadó, Budapest, 1995.

3. Bertóti, E.: Complementary energy method for cylindrical shells using second order stress functions, Computer Methods in Applied Mechanics and Engineering 1993; 108:147-163.

4. Bertóti, E.: Non-linear shell theory in terms of stresses and rotations, DSc thesis, Hungarian Academy of Sciences - University of Miskolc, 2003, p. 133 (in Hungarian).

5. Brezzi, F., Fortin, M.: Mixed and hybridfinite element methods, Springer Verlag, New York, 1991.

6. Fraeiss de Veubeke, B.M. A new variational principle for finite elastic displacements, International Journal of Engineering Sciences 1972; 10:745-763.

7. Hellinger, E.: Der allgemeine Ansatz der Mechanik der Kontinua, Encyclopädie der Matematischen Wissenschaften 1914; 4:602-694.

8. Naghdi, P. M.: Foundations of Elastic Shell Theory, In: Progress in Solid Mechanics, Volume IV (I. N. Sneddon and R. Hill, Eds.), North-Holland Publishing Company, Amsterdam, 1963, pp. 1-90.

9. Reissner, E.: On a variational theorem in elasticity, Journal of Mathematics and Physics 1950; 29:90-95.

10. ReIssner, E.: A note on variational principles in elasticity, International Journal of Solids and Structures 1965; 1:93-95 